\title{
Exploring the Interplay of Resilience and Energy Consumption for a Task-Based Partial Differential Equations Preconditioner
}

\author{
F.Rizzi ${ }^{\mathrm{a}, *}$, K.Morris $^{\mathrm{a}}$, K.Sargsyan ${ }^{\mathrm{a}}$, P.Mycek ${ }^{\mathrm{b}}$, C.Safta $^{\mathrm{a}}$, O. Le Maître ${ }^{\mathrm{c}}$, \\ O.M. Knio ${ }^{\mathrm{b}}$, B.J. Debusschere ${ }^{\mathrm{a}}$ \\ ${ }^{a}$ Sandia National Labs, Livermore, CA, USA \\ ${ }^{b}$ Duke University, Durham, NC, USA \\ ${ }^{c}$ LIMSI, Orsay, France
}

\begin{abstract}
We discuss algorithm-based resilience to silent data corruptions (SDCs) in a task-based domain-decomposition preconditioner for partial differential equations (PDEs). The algorithm exploits a reformulation of the PDE as a sampling problem, followed by a solution update through data manipulation that is resilient to SDCs. The implementation is based on a server-client model where all state information is held by the servers, while clients are designed solely as computational units. Scalability tests run up to $\sim 51 K$ cores show a parallel efficiency greater than 90\%. We use a 2D elliptic PDE and a fault model based on random single and double bit-flip to demonstrate the resilience of the application to synthetically injected SDC. We discuss two fault scenarios: one based on the corruption of all data of a target task, and the other involving the corruption of a single data point. We show that for our application, given the test problem considered, a four-fold increase in the number of faults only yields a $2 \%$ change in the overhead to overcome their presence, from $7 \%$ to $9 \%$. We then discuss potential savings in energy consumption via dynamic voltage/frequency scaling, and its interplay with fault-rates, and application overhead.

Keywords: Resiliency, server-client programming model, dynamic voltage/frequency scaling, PDE, domain-decomposition, silent data corruption.
\end{abstract}

${ }^{*}$ Corresponding author: fnrizzi@sandia.gov

Preprint submitted to Journal of ${ }^{A} T_{E} X$ Templates

March 31, 2017 


\section{Introduction}

The evolution of computing platforms towards exascale presents key challenges related to resiliency, power, memory access, concurrency and heterogeneous hardware 1, 2, 3, 4, 5. There is no consensus on what a "typical" exascale 5 architecture might look like 2. One of the main concerns is understanding how hardware will affect future computing systems in terms of reliability, energy consumption, communication and computational models.

The main constraint to making exascale computing a reality is energy consumption [4. The current target is to build an exascale machine consuming $\sim 20 M W$ by 2020 . Significant technological advances are required to make this objective feasible, since current systems cannot be simply scaled up to reach this goal. These advancements need to span different hardware aspects, ranging from underlying circuits, to power delivery as well as cooling technologies. Hardware-oriented research should be complemented by cross-cutting efforts tackling energy efficiency at the algorithm and programming model level. There is consensus that a coordination of efforts is required between advances in programming systems and the development of hardware to enable applications to run efficiently and correctly on exascale machines [1, 3].

Exascale simulations are expected to rely not only on thousands of CPU cores running up to a billion threads, but also on extensive use of accelerators, e.g. Graphics Processing Units (GPUs) [1, 3, 5]. This framework will necessarily lead to systems with a large number of components. The presence of many components, the variable operational modes (e.g. lower voltage to address energy requirements) and the increasing complexity of these systems (e.g. more and smaller transistors) can become a liability in terms of system faults. Exascale systems are expected to suffer from errors and faults more frequently than the current petascale systems [6, 5. Current parallel programming models and applications will require a resilient infrastructure to be suitable for fault-free simulations across many cores for reasonable amounts of time. It will become 
increasingly more important to develop resilient-aware applications for exascale, where fault-tolerance is explored and quantified to assess whether or not they can tolerate expected failure rates.

Energy and resilience are tightly linked challenges. For instance, high resilience could be achieved through extensive hardware redundancy, but this 35 approach would yield a large power overhead, e.g. three times more expensive for triple-redundancy. Checkpointing is currently the approach most widely used to recover from faults, but it is expected to become unfeasible for exascale applications given the higher failure rates [3, 5]. To address resilience without an excess power and/or performance costs will require innovations and coor40 dinated efforts across all system levels. At the application level, one approach would be to design applications such that they are structured into stages having different resilience requirements. This would allow one to isolate those data and computational units requiring resilience from other data and work units where resilience is less needed.

This work presents a new task-based resilient domain-decomposition partial differential equation (PDE) preconditioner implemented with a server-client programing model. The problem is reformulated such that the PDE solver is reduced to a number of independent tasks to benefit concurrency and parallelism. The algorithm enables the application to be resilient to silent data corruption ${ }_{50}$ (SDC), while the server-client model (SCM) enables resiliency to hard faults. Our implementation uses the User Level Fault Mitigation MPI (MPI-ULFM) [7, a fault tolerance capability proposed for the MPI standard that enables a fault-tolerant MPI framework. In this work, however, we don't focus on hard faults, whose analysis will be the subject of a separate study, but limit our attention to SDCs. Our application can be seen as a preconditioner that will enable today's solvers to be used effectively on future architectures by operating on subdomain levels. Scalability tests run up to $\sim 51 K$ cores show a parallel efficiency greater than $90 \%$.

The server-client programming model provides a task-based application with an infrastructure that can potentially address some of the concerns related to en- 
ergy consumption and resiliency. The work we present here assumes a SCM running on a machine with different capacity cores assigned to servers and clients. The idea pushed forward is that high-end high-capacity/voltage/reliability nodes are reserved for the servers which hold all the state information of the application, while lower-voltage higher-fault-rate components are used for clients which are in charge of the computation. This separation of data and computation enables the overall reduction of energy consumption for large scale machines, provided that the number of nodes hosting the servers is negligible compared to that hosting the clients, and the overhead associated with clients with higher fault rates is sufficiently small.

The paper is organized as follows. In $\S 2$ we describe the mathematical formulation; in $\S 3$, we present the implementation details; in $\S 4$ we discuss the results, focusing on the scalability $\S 4.1$, and resilience $\S 4.2$, in $\S 5$ we analyze the interplay between energy and resilience. Finally, $\S 6$ presents the conclusions.

\section{Mathematical Formulation}

We present the formulation for a generic 2D elliptic PDE of the form

$$
\mathcal{L} y(\mathbf{x})=g(\mathbf{x}),
$$

where $\mathcal{L}$ is an elliptic differential operator, $g(\mathbf{x})$ is a given source term, and $\mathbf{x}=\left\{x_{1}, x_{2}\right\} \in \Omega \subset \mathbb{R}^{2}$, with $\Omega$ being the target domain region. We focus on

so Dirichlet boundary condition $\left.y(\mathbf{x})\right|_{\mathbf{x} \in \Gamma}=y_{\Gamma}$ along the boundary $\Gamma$ of domain $\Omega$. A formulation of the algorithm focusing on $1 D$ elliptic PDEs can be found in [8]. Elliptic equations are chosen as test case because they are a fundamental problem in physics.

Figure 1 shows a high-level schematic of the algorithm's workflow. The starting point is the discretization of the computational domain. In general, the choice of the discretization method is arbitrary, potentially heterogeneous across the domain, e.g. uniform, or non-uniform rectangular grid, or a finite-element triangulation, etc. 


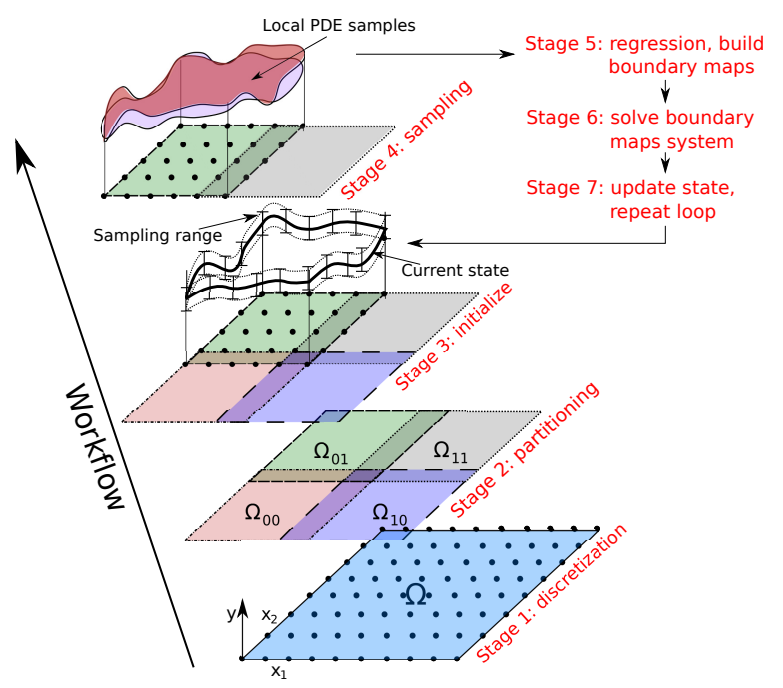

Figure 1: Schematic of the workflow of the algorithm. For clarity, starting with stage 2 we only show the steps for $\Omega_{01}$ but the same "operations" are applied to all subdomains.

The second step is the partitioning stage. The target $2 \mathrm{D}$ domain, $\Omega$, is partitioned into a grid of $n_{1} \times n_{2}$ overlapping regions (or subdomains), with $n_{k}$ being the number of subdomains along the $x_{k}$-th axis. The size of the overlap does not need to be equal and uniform among all partitions, and can vary across the domain. The partitioning stage yields a set of $n_{1} \times n_{2}$ subdomains $\Omega_{i j}$, and their corresponding boundaries $\Gamma_{s_{i j}}$, for $i=0, \ldots, n_{1}-1$, and $j=0, \ldots, n_{2}-1$, where $\Gamma_{s_{i j}}$ represents the boundary set of the $i j$-th subdomain $\Omega_{i j}$.

We define as our object of interest the set of solution fields along the boundaries, which we denote $\left.y(\mathbf{x})\right|_{\mathbf{x} \in \Gamma_{s_{i j}}}$ for $i=0, \ldots, n_{1}-1$, and $j=0, \ldots, n_{2}-1$. Due to the overlapping, each subdomain $\Omega_{i j}$ includes inner boundaries, $\Gamma_{s_{i j}}^{i n}$, i.e. the parts of the boundaries contained within $\Omega_{i j}$ that belong to the intersecting (neighboring) subdomains. The core of the algorithm relies on exploiting within each subdomain $\Omega_{i j}$ the map relating the solution at the subdomain boundaries, $\left.y(\mathbf{x})\right|_{\mathbf{x} \in \Gamma_{s_{i j}}}$, to the solution along the inner boundaries, $\left.y(\mathbf{x})\right|_{\mathbf{x} \in \Gamma_{s_{i j}}^{i n}}$. These maps can be written compactly as

$$
\left.y(\mathbf{x})\right|_{\mathbf{x} \in \Gamma_{s_{i j}}^{i n}}=f^{(i j)}\left(\left.y(\mathbf{x})\right|_{\mathbf{x} \in \Gamma_{s_{i j}}}\right),
$$


for $i=0, \ldots, n_{1}-1$, and $j=0, \ldots, n_{2}-1$. The system of equations assembled

from these boundary-to-boundary maps collected from all subdomains, combined with the boundary conditions on the full domain $\left.y(\mathbf{x})\right|_{\mathbf{x} \in \Gamma}$, yields a fixed-point problem of the form

$$
\mathbf{y}(\mathbf{x})=\mathcal{F} \mathbf{y}(\mathbf{x})
$$

where $\mathbf{y}$ represents the vector of the solution values at all subdomains boundaries. This problem is only satisfied by the true solution. We remark that these boundary maps $f^{(i j)}$ relate the $y$-values, since they are built from the restrictions of the subdomain solutions at the corresponding boundaries.

In this work, rather than solving Eq. 3 directly, we construct approximations (or surrogates) of the boundary-to-boundary maps, which we call $\tilde{f}^{(i j)}$. One of the main features of the algorithm is that the construction of these maps can be done for each subdomain independently from all the others. This allows us to satisfy data locality which is key to achieve scalability on extreme scale machines. To build these surrogate maps, given a current "state" of the solution at the subdomains boundaries, we use a sampling strategy that involves solving the target PDE equation locally within each subdomain for sampled values of the boundary conditions on that subdomain, see stage 3 in Figure 1 . These samples are used within a regression approach to "infer" the approximate boundary-toboundary maps. In general, for non-linear problems the maps are non-linear, while for linear PDEs the boundary maps are linear [8]. Following the construction of the surrogate boundary-to-boundary maps, we can then solve the approximate version of the fixed point system in Eq. (2), which provides us with the new solution state at all the subdomains boundaries and represents an approximation of the true solution. An important measure of the accuracy of the current solution $\left.y(\mathbf{x})\right|_{\mathbf{x} \in \Gamma_{s_{i j}}}$ is the residual vector, defined as

$$
\mathbf{z}=\mathcal{F} \mathbf{y}-\mathbf{y}
$$

which can be computed by extra subdomain solves using boundary conditions defined by the current solution $\mathbf{y}$, and subtracting the corresponding current solutions $\mathbf{y}$ from the resulting values at all boundaries. Given the fixed-point 
problem in Eq. (3), the residual (4) vanishes if the current solution $\mathbf{y}$ is the exact solution. In the case of linear PDEs, because the boundary-to-boundary maps are linear, and assuming that all the regressions complete successfully, the algorithm converges in one iteration.

The construction of the boundary-to-boundary maps plays a key role for ensuring resilience against potential silent data corruption (SDC) affecting the PDE samples. As shown in [8, when inferring linear maps, using a $\ell_{1}$-noise model one can seamlessly filter out the effects of few corrupted data. The $\ell_{1}$ noise model yields the solution with as few non-zero residuals as possible. Under the assumption that faults are rare, the inferred maps will fit the noncorrupted data exactly while effectively ignoring the corrupted data. In the present work, we employ an iteratively re-weighted least squares (IRLS) method, which is effectively equivalent to a $\ell_{1}$ minimization [9]. Figure 2 shows a test proving the resilience of the regression stage. The PDE used to generate these results is described later in the results section. Panel (a) shows sample PDE solutions generated over a target subdomain for sampled boundary conditions. We synthetically corrupt two samples using a random bit flip: in one case, shown by the green surface, we corrupt one boundary condition point before the task execution; for the second corrupted sample, shown by the red surface, we corrupt a single point in the inner grid after the task execution. These two types of corruptions can both be categorized as SDC, because they do not cause the termination of the application.

From the PDE samples above, we collect the subset obtained at a test inner location $\mathbf{x}_{*}=(0.3,0.6)$ (shown as a blue circle in the figure), and infer the approximate linear map $y_{*}(\mathbf{x})=c_{0}+\sum_{i=1}^{N} c_{i} y_{i}(\mathbf{x})$, where $i$ enumerates the points along the subdomain perimeter. For this test, we generated a total of 30 PDE samples: 25 is the minimum number to have a well-posed linear regression given the size of the subdomain $(N=24$ for this specific case), while 5 additional samples are added to guard against possible faulty data. We perform the regression using both the $\ell_{1}$ and $\ell_{2}$ models, and report in panel (b) the error between the approximate map and the one obtained using the uncorrupted 


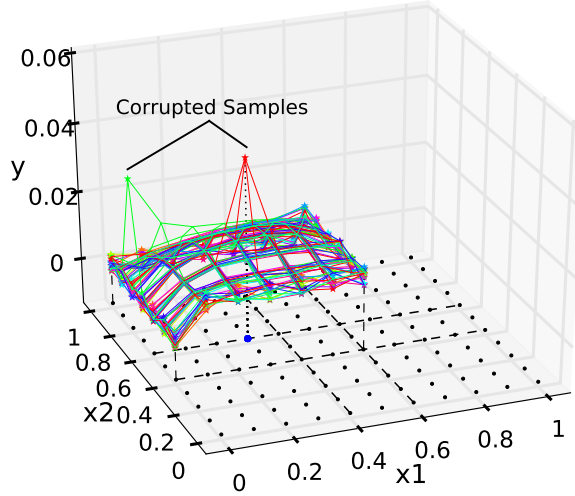

(a)

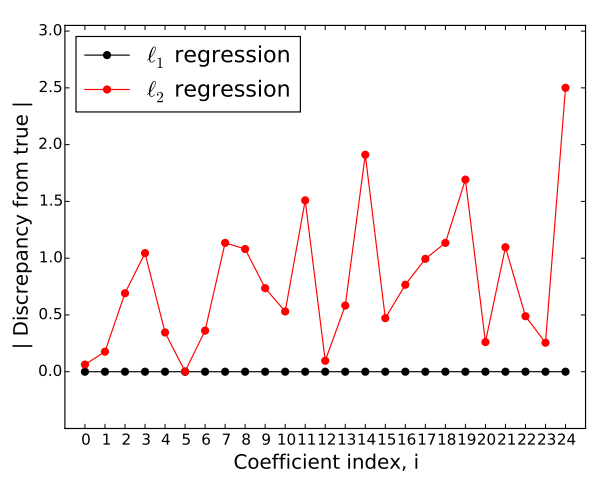

(b)

Figure 2: Simple test proving the resilience of the $\ell_{1}$ regression using the PDE described later in the results section. Panel (a) shows sample PDE solutions generated over a target subdomain for sampled boundary conditions. Panel (b) shows the distance of the approximate map obtained through $\ell_{1}$ and $\ell_{2}$ regressions, to the "true" map for the target blue point in (a). The "true" map is obtained using regression with uncorrupted samples, while the approximate map uses the data presented in (a), where two samples are corrupted.

samples. The results show that the $\ell_{1}$-based regression matches exactly the uncorrupted result, being completely unaffected by the corrupted data points. On the contrary, using $\ell_{2}$ yields the wrong answer since the corrupted data have substantial effect. The key underlying point demonstrated is that even in the presence of corrupted PDE samples, provided we have enough samples, we do not need to waste resources and energy to identify them in order to have a successful regression. The correctness of the result is ensured by the mathematical properties of the regression model.

\section{Implementation Details}

We have developed a parallel, $\mathrm{C}++$ implementation of the algorithm using a server-client model (SCM). This section describes the SCM, its resilience properties, and how we implement each stage of the algorithm to exploit the SCM model. 


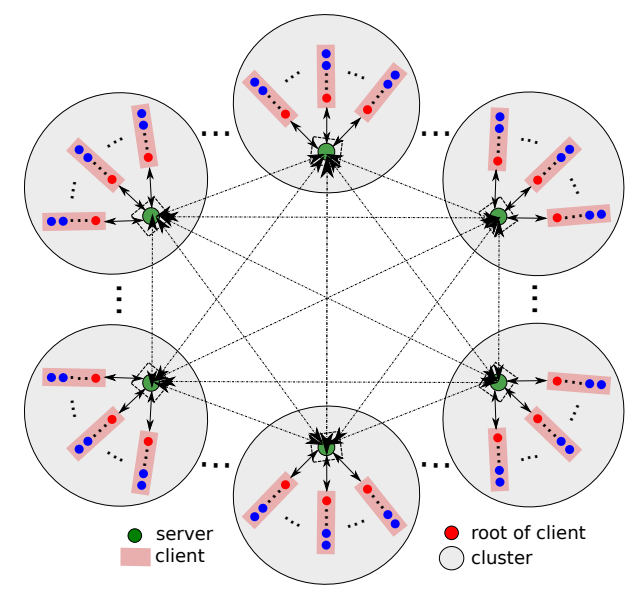

Figure 3: Schematic of the server-client structure.

\subsection{Server-Client Model}

Figure 3 shows a schematic of our SCM structure. We adopt a cluster-based model where the MPI ranks are grouped into separate clusters, with each cluster containing a server and, for resource balancing purposes, the same number of clients. All servers can communicate between each other, while the clients within a cluster are only visible to the server within that cluster. Moreover, within any given cluster, clients are independent, i.e. at a given time instant, each client is handling a different work unit and they cannot communicate with each other. This design choice allows a client to fail without affecting other clients. Only the work being executed by the failed client is affected.

The data is distributed among the servers, and these are assumed to be highly resilient (safe or under a "sandbox" model implementation). The sandbox model assumed for the servers can be supported by either software or hardware. In the case of software support, this can be accomplished by a programming model relying on data redundancy and strategic synchronization [10, 11, 12. The latter assumption is supported by hardware specifications on the variable levels of resilience that can be allowed within large computer systems.

Since the servers hold the data, they are responsible for generating work in the form of tasks, dispatching them to their pool of available clients, as well as 


\subsection{Algorithm Implementation}

The algorithm described in $\S 2$ involves four main stages: sampling, regression, fixed-point solve, and updating. As mentioned before, sampling and regres- 
This feature reveals their task-based nature, and are therefore implemented in the form of tasks executed by the clients. As such, they are vulnerable to the failures occurring on the clients. On the other hand, the fixed-point solve of the boundary-to-boundary maps system and the updating of the subdomains are safely executed by the servers, since they fully own the state information. The system of equations built from the boundary maps is much smaller than original discretized PDE system over the full domain grid, and so it fits on a small number of servers. Moreover, the servers are assumed to be "sandboxed", allowing us to circumvent any potential data corruption during these operations. This $\mathrm{PDE}$

$$
\frac{\partial}{\partial x_{1}}\left(k(\mathbf{x}) \frac{\partial y(\mathbf{x})}{\partial x_{1}}\right)+\frac{\partial}{\partial x_{2}}\left(k(\mathbf{x}) \frac{\partial y(\mathbf{x})}{\partial x_{2}}\right)=g(\mathbf{x}),
$$

where $\mathbf{x}=\left\{x_{1}, x_{2}\right\}$, the field variable is $y\left(x_{1}, x_{2}\right), k\left(x_{1}, x_{2}\right)$ is the diffusivity, and $g\left(x_{1}, x_{2}\right)$ is the source term, over the unit square $(0,1)^{2}$ with homogeneous Dirichlet boundary conditions. The diffusivity and source fields are defined as

$$
\begin{aligned}
& k\left(x_{1}, x_{2}\right)=8.0 * \exp \left(-d\left(x_{1}, x_{2}\right) / 0.025\right)+2.0 \\
& g\left(x_{1}, x_{2}\right)=2.0 * \exp \left(-d\left(x_{1}, x_{2}\right) / 0.050\right)-1.0
\end{aligned}
$$

where $d\left(x_{1}, x_{2}\right)=\left(x_{1}-0.35\right)^{2}+\left(x_{2}-0.35\right)^{2}$. To solve the above PDE within each subdomain, we employ a structured grid and second-order finite differences to discretize Eq. (5). The resulting linear system stemming from the finite-difference discretization is solved using the parallel solver AztecOO in Trilinos 16. 
Table 1: Scalability parameters.

\begin{tabular}{lc}
\hline & Weak Scaling Parameters \\
\hline \hline Subdomains & $12^{2}, 18^{2}, 24^{2}, 30^{2}, 36^{2}, 42^{2}$ \\
\hline Subdomain grid size & $180^{2}$ \\
\hline Num. of Servers & $16,36,64,100,144,196$ \\
\hline Num. of clients/server & 64 \\
\hline Size of client & 4 MPI ranks \\
\hline Total Cores & $9252,12594,16448,25700,37008,50372$ \\
\hline
\end{tabular}

\subsection{Nominal Scalability}

We tested the scalability of our application on Edison at NERSQ1 a Cray XC30, with Peak performance of 2.57 Petaflops, Cray Aries high-speed interconnect with Dragonfly topology with approximately $\sim 8 \mathrm{~GB} / \mathrm{sec}$ MPI bandwidth. Table 1 lists the parameters used for the scalability runs. These runs use the Edison's native Cray-MPICH.

The weak scaling is setup by fixing the number of clients per server and the amount of data owned by each server, and increasing the problem size by adding increasingly more clusters, as shown in Table 1. This design imposes no constraint on the problem size, since larger problems can be tackled by simply adding more clusters. Figure 4 presents the results up to $\sim 51 \mathrm{~K}$ cores, specifically highlighting the scaling of the most important stages of the algorithm, as well as the scaling of the full application. The results show an excellent behavior for each individual stage and for the full application, with efficiency above $90 \%$.

\subsection{Resiliency}

This section describes and demonstrates the resilience properties of our PDE solver, specifically focusing on resilience to SDC. We evaluate the resilience against SDC affecting numerical data used in the algorithm, i.e. we exclude

1 http://www.nersc.gov 


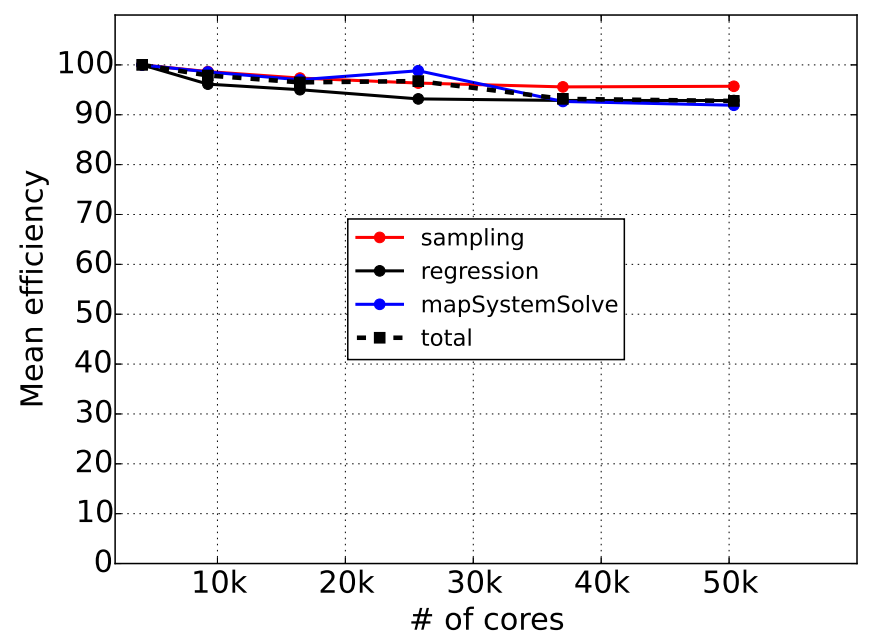

Figure 4: Nominal weak scaling results: the mean efficiency is defined as $t_{\text {ref }} / t * 100$, where $t_{r e f}$ is the execution time for the smallest case.

other types of faults e.g., in data structures or control flow, since these issues represent a different problem [17]. We model SDC as bit-flips, and the results below are based on a random bit-flip model to inject the faults. Specifically, we explore the case where a SDC is caused by a single random bit-flip, as well as two random bits being flipped. The latter is more rare than the former, but also more dangerous because current technology like ECC can only handle single bit-flips [18]. Contrary to the work by [17, 19], we do not characterize only the effect of the worst case scenario, or very outrageous faults. We believe, in fact, that in many scientific applications, the biggest problems might not come from the occurrence of an outrageous fault, but from a small corruption in the data at some point during the simulation. One example is scientific simulations that tie to chaos theory, like climate models and/or turbulence: in such cases, the simulations are very sensitive to, e.g., initial conditions, where even small variations of the initial data can yield large deviations in the model predictions. As will be shown below, in fact, this is the case for the present algorithm. When an outrageous fault occurs, it is easily filtered out. However, when a more subtle corruption occurs, the solver takes more effort to converge 
towards the right solution. The variability of corruptions possible by using a bit flip model is a useful resource allowing us to test and assess our applications under all these various scenarios.

Test Problem and Execution

As a test problem for resilience, we adopt the PDE introduced in Eq. (5), solved over a structured uniform grid with $201^{2}$ grid points over the unit square domain. We partition the domain using $n_{1}=n_{2}=4$ subdomains, with an overlapping of 4 grid cells between neighboring subdomains. This setting yields 290 a total of 16 subdomains, each with a local grid of $54^{2}$ grid points. Nominally, this problem involves $N_{\text {nom }}^{s}=3408$ sampling tasks, and $N_{n o m}^{r}=2496$ regression tasks. The SCM structure involves a single server holding the data, i.e. subdomains, and uses 14 clients each with size 2 .

\section{Fault Injection}

295 Our goal is to evaluate how the application behaves as we increase the number of faults. We leverage the task-based nature of our algorithm by choosing the number of faults to inject as a percentage of the nominal number of tasks to execute. Since we know in advance how many tasks are needed by our test problem, we randomly select off-line the set of task IDs that will be hit by a fault during the execution. One advantage of this method is that the number of faults hitting the system is well-known, and it eliminates any dependency between faults occurrence and the execution time, since the latter is machinedependent. Moreover, if needed, this setting still allows us to extract an average fault rate, given a total execution time and the known number of faults. We explore various levels of corruption, namely $0.25 \%, 0.5 \%, 0.75 \%$, and $1.0 \%$ of the nominal number of tasks. For each percentage of corruption, we run 150 runs to have a statistically meaningful result.

Exploiting a selective reliability approach [15, 17, 20, 19, which lets algorithm developers isolate faults to certain parts of the algorithm, in this paper we focus on the results obtained by injecting the faults during the sampling 
stage only. The results obtained for the other scenarios, e.g. involving faults hitting the regression are left for future extensions. Faults are only injected in the clients, consistently with the SCM described previously, where servers are assumed to be reliable, while no assumption is made on the reliability of the clients.

\section{Fault Model}

In this work, we analyze two cases: one involves corrupting all elements in the array holding boundary conditions contained in the task, while the other involves corrupting only a single array element. The reason behind this distinction is that large, it is likely that at least one large bit (e.g. exponent) is flipped, causing the value to become "outrageous". One the contrary, when a single data variable is corrupted, the likelihood of flipping a bit in the exponent is lower. We believe that both scenarios are important to examine, since they provide information on the algorithm's sensitivity to different levels of data corruption.

When a fault needs to be injected, we adopt the following procedure: we draw a value, $u$, from a standard uniform distribution, and if $u \leq 0.5$, the task data is corrupted before the execution; if $u>0.5$, the task data is corrupted after its execution. This allows us to mimic corruptions that occur when tasks are being transmitted to and from a client, as well as those happening during execution. If a task is corrupted before the execution, this translates into corrupting all or a single point in the boundary conditions owned by that sampling task, since the boundary conditions are the only information needed to run a solve of the elliptic PDE. If a task is corrupted after execution, this translates into corrupting all or a single point in the solution, which means that even the inner points of a subdomain can be affected.

\section{Handling Faults}

Given the fault model described above, several fault scenarios unfold. The mechanisms that we incorporate in the algorithm to make it resilient are kept to 

algorithm towards faults in the sampling, the key condition to be satisfied is that out of the samples used in the regression, the number of uncorrupted samples has to be greater than the minimum set needed to have a mathematically wellposed regression problem. To this end, we mitigate the impact of faults in minimum set. This is accomplished by defining an oversampling factor, $\rho>1$, such that the target number of samples to generate is $N=\rho N_{n o m}^{s}$. Moreover, during the sampling stage, we apply a filter on the task data returned to the server to check that it is within the interval $(-100,100)$ before the data is stored within the corresponding subdomain. We anticipate that due to the random bit-flip mode, this interval has been chosen such that number of cases falling outside is small compared to those falling inside. This interval is arbitrary, but can be estimated by either a domain expert or by known physical bounds on the solution. In general, this bound does not have to tight, but obviously the tighter data, then those could be easily used to derive some bounds. Otherwise, one can resort to some theoretical analysis to derive bounds for the PDE solution under study. This is the only active "filter" that is needed by the application. Any other corruption during the sampling does not need to be actively detected, since it is seamlessly filtered out thanks to the mathematical model used in the regression as shown in Figure 2.

\subsubsection{Resilience Analysis}

This section focuses on the resilience results obtained for the runs described above. Figure 5 summarizes the main statistics from the ensemble runs performed for the single bit-flip scenario. Specifically, we show four radar-plots, corresponding to the following four different combinations: first row shows the results when all data in a sampling task is corrupted, with $\rho=1.1$ (a) and $\rho=1.07$ (b); second row shows results for single data point corruption in a sampling task, with $\rho=1.1$ (c) and $\rho=1.07$ (d). Each radar-plot displays the 


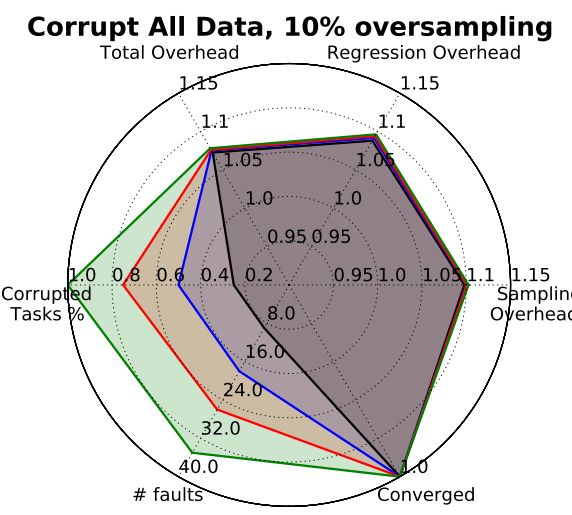

(a)

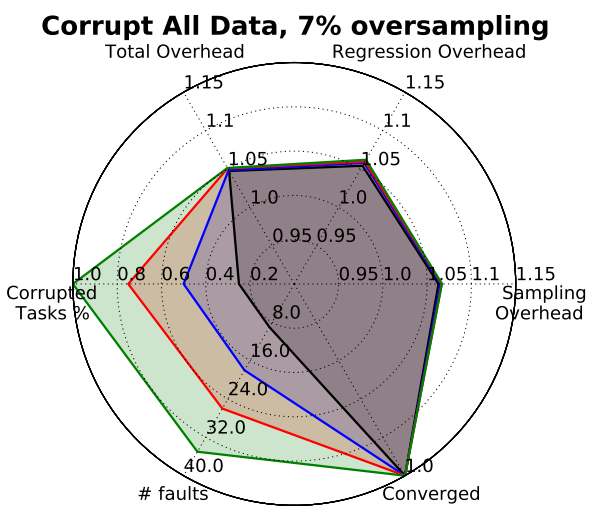

(b)

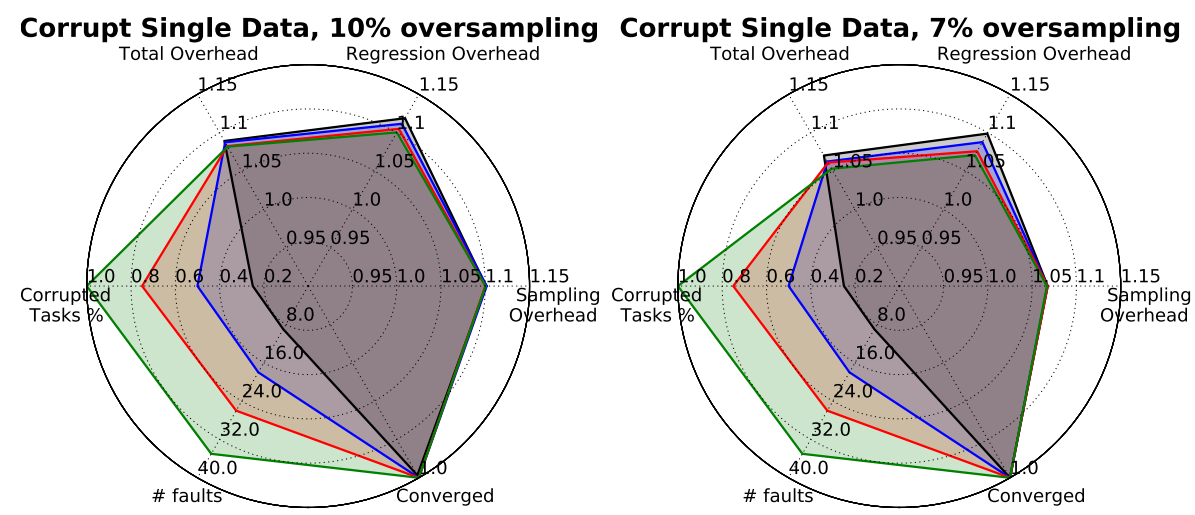

(c)

(d)

Figure 5: Statistical results obtained from the ensemble runs performed for the resilience analysis. The radar-plots correspond to the following four cases: first row shows results when all data in a sampling task is corrupted, with $\rho=1.1$ (a) and $\rho=1.07$ (b); second row shows results for single data point corruption in a sampling task, with $\rho=1.1$ (c) and $\rho=1.07$ (d).

average value over 150 replicas of six key quantities in the following clock-wise order: the total overhead of the application runtime compared to the no-fault runs, the regression and sampling overhead, the boolean value identifying convergence, the number of tasks affected by a fault, and the percentage of tasks corrupted. In all cases, the runs converge to the correct answer. Convergence is verified by checking that the root-mean-square error computed for the residual in Eq. (4) is below a specified threshold of $10^{-13}$. This proves that the 
application is resilient to faults occurring during sampling.

For a fixed value of $\rho$, the plots show that the overhead with respect to the no-fault case is smaller when all the data in a sampling task is corrupted. that the differences are minimal, demonstrating that the algorithm formulation and the underlying $\ell_{1}$ regression model used make the application minimally sensitive to the number of bit-flips occurring. 
Table 2: Overhead comparison between single bit-flip (SBF) and double bit-flip (DBF) corruption for the case where a single data point is corrupted and $7 \%$ oversampling.

\begin{tabular}{ccc|cc|cc|cc}
\hline Corrupted tasks $\%$ & \multicolumn{2}{c|}{$0.25 \%$} & \multicolumn{2}{|c|}{$0.5 \%$} & \multicolumn{2}{|c|}{$0.75 \%$} & \multicolumn{2}{c}{$1.0 \%$} \\
\hline & SBF & DBF & SBF & DBF & SBF & DBF & SBF & DBF \\
\hline $\begin{array}{c}\text { Regression } \\
\text { Overhead (\%) }\end{array}$ & 1.099 & 1.111 & 1.088 & 1.092 & 1.076 & 1.085 & 1.071 & 1.071 \\
\hline $\begin{array}{c}\text { Total } \\
\text { Overhead (\%) }\end{array}$ & 1.070 & 1.075 & 1.062 & 1.069 & 1.061 & 1.063 & 1.053 & 1.054 \\
\hline
\end{tabular}

\section{Trade-off between Energy and Resilience}

In this section, we discuss how the resilience properties of our application allow us to draw conclusions about potential savings in the energy consumption. Resilience and energy consumption are tightly linked 21, 22. It has been shown that voltage decrease is linked to higher faults rates, see [21] and references therein. We demonstrate below how lowering the energy consumption during the sampling stage by means of voltage scaling allows us to save energy and still run the application successfully despite being affected by more frequent system faults. This framework can be enabled because of the SCM, which allows us to separate state from computation. Decreasing the energy consumption is possible via variable-voltage CPUs, which can reduce power consumption quadratically at the expense of linearly reduced speed 21 . The reason for this is that circuit delay is almost linearly related to $1 / V$, where $V$ is the voltage, so for systems to function correctly, the operating frequency needs to decrease linearly to the decrease of the supply voltage.

We compare two scenarios: (A) involves running the application assuming that all machine components run at full operational capacity/speed; (B) is based on decreasing the energy consumption of the clients only during the sampling stage by reducing the operational efficiency of the corresponding processing units. Moreover, to compare the two scenarios, they tackle the same problem, have the same number of servers and clients, are run on the same machine. 
The servers always run at full capacity to keep the state safe. We remark that the only difference between the two cases lies in how the sampling stage is run. The other stages of the algorithm are equivalent. The analysis below is carried out without accounting for the energy consumed by the network and/or system cooling. We are aware that the energy consumption for these HPC systems' components contributes to the total energy budget, but we limit the focus of this work to highlighting the relationship between system faults, algorithmic resilience and computing energy.

To lay the ground of the analysis, we define how to estimate the energy consumption following the work in 21,22 . In embedded systems, the power is consumed mainly by the processor, memory, clock and underlying circuits. The power consumption, $P$, during activity can be modeled as [21]:

$$
P=\hat{P}+C V^{2} f
$$

where $\hat{P}$ is the frequency independent active power (independent of frequency and voltage), $C$ is the switch capacitance, $V$ is the voltage, and $f$ is the frequency. Note that as in [21, we have neglected the contribution of the sleep power since it does not have any effect on the energy savings and we assume the system to be always on. The energy consumed by an operation running over the time interval $T=t_{2}-t_{1}$ is then

$$
E=\left(\hat{P}+C V^{2} f\right) T
$$

We now proceed by estimating the energy consumption of our application in 445 both scenarios. The nominal (or full energy) scenario involves clients operating at maximum voltage, $V_{m}$, and frequency, $f_{m}$, and includes $N_{1}$ sampling tasks, with each task execution taking a time $T_{1}$. For this reference scenario, the total energy consumed by the clients to execute $N_{1}$ samples is

$$
E_{1}^{s}=N_{1}\left(\hat{P} T_{1}+C V_{m}^{2} f_{m} T_{1}\right)
$$

For the reduced energy case, as mentioned above, both voltage and frequency are lowered to $V_{2}<V_{m}$, and $f_{2}<f_{m}$, such that $f_{m} / f_{2}=V_{m} / V_{2}$. This implies 


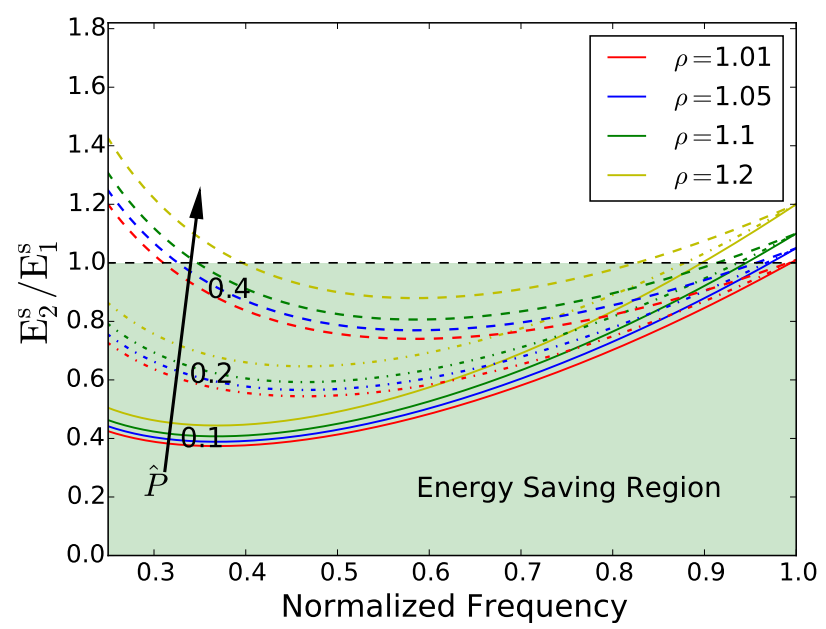

Figure 6: Energy ratio $E_{2}^{s} / E_{1}^{s}$ between the reduced, $E_{2}$, and full, $E_{1}$, case as a function of the normalized frequency. We show the curves obtained for $\hat{P}=\{0.1,0.2,0.4\}$, and varying oversampling factor $\rho=\{1.01,1.05,1.1,1.2\}$. (Frequencies below 0.25 are not shown assuming 0.25 to be a reasonable value for the lowest operational frequency of a processor.)

that the execution time of a task is $T_{2}=T_{1} \frac{f_{m}}{f_{2}}$, as it depends linearly on the frequency. This assumption is, in general, valid for compute-intensive tasks. Due to the interplay between voltage and reliability, we expect for this lowenergy scenario a higher probability of faults occurring during the sampling. We mitigate the effect of these faults by generating more samples, i.e. we assume $N_{2}=\rho N_{1}$, where $\rho>1$ is the oversampling factor. This oversampling is needed for the algorithm, as shown in the resilience results from the previous section, to guard against potential data corruption. The total energy consumed by the clients to execute $N_{2}$ samples in the reduced energy case is

$$
\begin{aligned}
E_{2}^{s} & =N_{2}\left(\hat{P} T_{2}+C V_{2}^{2} f_{2} T_{2}\right) \\
& =N_{2}\left(\hat{P} T_{1} \frac{f_{m}}{f_{2}}+C V_{m}^{2} f_{m} T_{1} \frac{f_{2}}{f_{m}}\right),
\end{aligned}
$$

where Eq. 111 has been obtained by making some substitutions and rearranging terms to explicitly highlight the relationship with the nominal case. It is easy to see that the energy function has a minimum at $f_{2}^{*}=(0.5 \hat{P} / C)^{1 / 3}$. Below, we assume that voltages and frequencies are normalized, i.e. we set $V_{m}=1$ and 
$f_{m}=1$, such that any other voltage or frequency is in the interval $(0,1)$.

Figure 6 shows the ratio $E_{2}^{s} / E_{1}^{s}$ as a function of the frequency $f_{2}$. We show the results obtained for $\hat{P}=\{0.1,0.2,0.4\}$, and also vary the oversampling factor $\rho=\{1.01,1.05,1.1,1.2\}$. This figure allows us to make conclusion about how much energy we are able to save by leveraging the reduced-energy scenario as opposed to the full one. We can draw the following observations. First, the lower the value of $\hat{P}$, the more energy we are able to save. This is clear from Eq 11 since the contribution from $\hat{P}$ is proportional to the execution time. Second, the results show that as $\hat{P}$ decreases, the minimum of the energy curves is obtained for smaller and smaller frequencies. For the three cases shown, the optimal frequencies are $f_{2}^{*}=\{0.369,0.464,0.585\}$ for $\hat{P}=\{0.1,0.2,0.4\}$. Third, we see that for a given value of $\hat{P}$, the energy curves shift upward as the oversampling ratio $\rho$ increases. Intuitively, increasing $\rho$ increases the number of tasks to execute, and therefore we are able to save less and less energy. The results show, e.g., that if we assume $\hat{P}=0.4$, and run at the optimal frequency $f_{2}^{*}=0.585$, the energy saving ranges from $\sim 30 \%$ when $\rho=1.01$, to $\sim 15 \%$ when $\rho=1.2$. The frequency range shown in figure 6 was chosen to reveal the full trend of each curve for the selected values of $\hat{P}$ and $\rho$. But we remark that operating at the optimal (or too small) frequency is not always possible if this frequency is smaller than the minimum operational frequency, $f_{\text {low }}$, allowed by the processor, i.e. $f_{2}^{*}<f_{\text {low }}$, for a given voltage. To the best of our knowledge, 2 we think that a reasonable assumption would be $0.2<f_{\text {low }}<0.4$. The operational minimum energy efficient frequency that is feasible for a processor is $\max \left\{f_{\text {low }}, f_{2}^{*}\right\}$. Hence, the energy saved might be slightly smaller, but the plot shows that it is still considerable.

Beside increasing the execution time, voltage scaling also causes fault rates in the processors (including logic core and cache) to increase exponentially [21, 480 22, 23]. Assuming a Poisson process for the faults [21], the relationship between

\footnotetext{
${ }^{2}$ https://www.pugetsystems.com/labs/articles/Is-CPU-Base-Frequency-Still-a-RelevantSpec-512/
} 


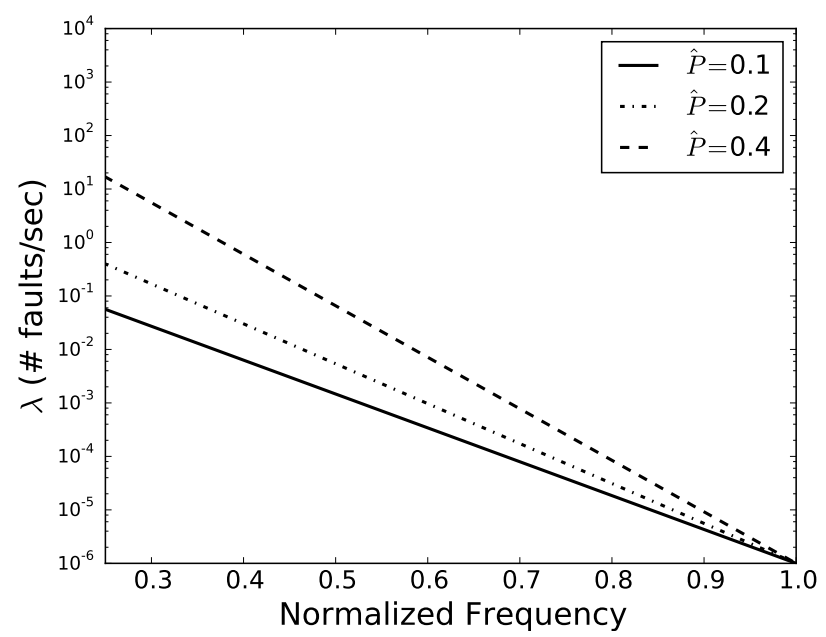

Figure 7: Fault rate, $\lambda$, as a function of the frequency, for three different values of $\hat{P}$, see Eq. 12 . For clarity, the $y$-axis is plotted in log-scale.

the fault rate, $\lambda$ (\# faults/sec), the voltage $V$ and frequency $f$ can be expressed as:

$$
\lambda=\lambda_{0} 10\left(d \frac{1-f}{1-\max \left\{f_{\text {low }}, f_{2}^{*}\right\}}\right),
$$

where $\lambda_{0}$ is the fault rate corresponding to $V_{m}$ and $f_{m}, d$ is a constant such that the larger its value, the more sensitive the fault rate is to scaling, and $f$ ${ }_{485}$ is the frequency corresponding to voltage $V$, such that $V_{m} / V=f_{m} / f$. Also, the dependence on $\hat{P}$ appears through the optimal frequency $f_{2}^{*}$, since $f_{2}^{*}=$ $(0.5 \hat{P} / C)^{(1 / 3)}$ as shown earlier. Similarly to [21], in this work we assume $\lambda_{0}=$ $10^{-6}$ (\# faults/sec), and $d=4$. We remark that the expression above might not be exactly applicable to current systems, but it is consistent with recent work on this topic, see e.g. 24]. The analysis below discusses the potential workflow that one would have to follow to calibrate the oversampling for tolerating an expected number of faults, it does not provide an exact formula for the dependence of the fault rate on the frequency.

Figure 7 shows how the fault rate varies as a function of the frequency for the three different values of $\hat{P}$ selected before. For clarity, the $y$-axis is plotted in log-scale. The first observation is that as the frequency decreases, the fault 
rate increases. From the figure, we can see that if the operating frequency is $f_{2}^{*}=\{0.369,0.464,0.585\}$, the corresponding expected fault rate is $\lambda=0.01$, namely about one fault every 100 seconds. The key question arising is: what is the trade-off between the energy saved due to voltage scaling and the energy spent to recover from the more frequent faults occurring due to lower operation power? This is where the resilience plays a key role, especially if the algorithm is inherently resilient to faults. If an application had a small overhead associated with recovering from faults, then most of the energy saved by running at reduced speed would be gained. On the other hand, if the overhead of the application to recover from a fault is substantial, then some of the energy saved by running at reduced speed would be offset, potentially eliminating any energy savings.

To calibrate a particular run, a user will have to choose a lower frequency $f_{2}$ to run the sampling stage, then use Figure 6 to determine the energy savings for a corresponding oversampling factor $\rho$. The expected fault rate can be computed using Eq. 12. The user must determine if the oversampling factor chosen is large enough to compensate for the expected number of faults and adjust the oversampling factor accordingly. If it is allowed by the machine, the target low-energy frequency $f_{2}$ should be chosen as close as possible to the optimal frequency $f_{2}^{*}$ to ensure that the energy saved during the sampling stage is not offset by the overhead of the regression stage.

\section{Conclusion}

We discussed algorithm-based resilience to silent data corruption (SDC) in a task-based domain-decomposition preconditioner for partial differential equa-

520 tions (PDEs).

The algorithm involves the following main steps: first, the domain of the PDE is split into overlapping subdomains; second, the PDE is solved on each subdomain for sampled values of the local current boundary conditions; third, the resulting subdomain solution samples are fed into a regression step to build boundary-to-boundary maps; finally, the intersection of these maps yields the 
updated state at the subdomain boundaries.

The implementation is based on a server-client model where all state information is held by the servers, while clients are designed solely as computational units. We tested weak scaling up to $\sim 51 K$ cores, showing an efficiency greater

\section{Acknowledgments}

This material is based upon work supported by the U.S. Department of Energy, Office of Science, Office of Advanced Scientific Computing Research, under Award Numbers 13-016717. Sandia National Laboratories is a multi-program 


\section{References}

[1] DOE-ASCR, Exascale programming challenges, Tech. rep. (Jul. 2011). URL http://science.energy.gov/ /media/ascr/pdf/ program-documents/docs/ProgrammingChallengesWorkshopReport. pdf

[2] J. A. Ang, R. F. Barrett, R. E. Benner, D. Burke, C. Chan, J. Cook, D. Donofrio, S. D. Hammond, K. S. Hemmert, S. M. Kelly, H. Le, V. J. Leung, D. R. Resnick, A. F. Rodrigues, J. Shalf, D. Stark, D. Unat, N. J. Wright, Abstract machine models and proxy architectures for exascale computing, in: Proceedings of the 1st International Workshop on Hardware-Software Co-Design for High Performance Computing, CoHPC '14, IEEE Press, Piscataway, NJ, USA, 2014, pp. 25-32. doi: 10.1109/Co-HPC.2014.4. URL http://dx.doi.org/10.1109/Co-HPC.2014.4

[3] K. Bergman, S. Borkar, D. Campbell, W. Carlson, W. Dally, M. Denneau, P. Franzon, W. Harrod, J. Hiller, S. Karp, S. Keckler, D. Klein, R. Lucas, M. Richards, A. Scarpelli, S. Scott, A. Snavely, T. Sterling, R. S. Williams, K. Yelick, K. Bergman, S. Borkar, D. Campbell, W. Carlson, W. Dally, M. Denneau, P. Franzon, W. Harrod, J. Hiller, S. Keckler, D. Klein, P. Kogge, R. S. Williams, K. Yelick, Exascale computing study: Technology challenges in achieving exascale systems peter kogge, editor \& study lead (2008). 
[4] DOE-ASCR, Top ten exascale research challenges, Tech. rep. (Feb. 2014).

585 [5] F. Cappello, A. Geist, W. Gropp, S. Kale, B. Kramer, M. Snir, Toward exascale resilience: 2014 update, Supercomputing frontiers and innovations 1 (1).

URL http://superfri.org/superfri/article/view/14

[6] F. Cappello, A. Geist, B. Gropp, L. Kale, B. Kramer, M. Snir, Toward Exascale Resilience, International Journal of High Performance Computing Applications 23 (4) (2009) 374-388.

[ [7] W. Bland, A. Bouteiller, T. Herault, G. Bosilca, J. Dongarra, Post-failure recovery of mpi communication capability: Design and rationale, Int. J. High Perform. Comput. Appl. 27 (3) (2013) 244-254. doi:10.1177/ 1094342013488238 .

URL http://dx.doi.org/10.1177/1094342013488238

[8] K. Sargsyan, F. Rizzi, P. Mycek, C. Safta, K. Morris, H. Najm, O. L. Matre, O. Knio, B. Debusschere, Fault resilient domain decomposition preconditioner for pdes, SIAM Journal on Scientific Computing 37 (5) (2015) A2317-A2345. doi:10.1137/15M1014474.

[ [9] I. Daubechies, R. DeVore, M. Fornasier, C. S. Güntürk, Iteratively reweighted least squares minimization for sparse recovery, Communications on Pure and Applied Mathematics 63 (1) (2010) 1-38. doi:10.1002/cpa. 20303

URL http://dx.doi.org/10.1002/cpa.20303

[10] M.-L. Li, P. Ramachandran, S. K. Sahoo, S. V. Adve, V. S. Adve, Y. Zhou, n Understanding the propagation of hard errors to software and implications for resilient system design, SIGOPS Oper. Syst. Rev. 42 (2) (2008) 265276. doi:10.1145/1353535.1346315.

URL http://doi .acm.org/10.1145/1353535.1346315 
[11] P. G. Bridges, K. B. Ferreira, M. A. Heroux, M. Hoemmen, Fault-tolerant linear solvers via selective reliability, ArXiv e-printsarXiv:1206.1390.

[12] C. Engelmann, T. Naughton, Toward a performance/resilience tool for hardware/software co-design of high-performance computing systems, in: Parallel Processing (ICPP), 2013 42nd International Conference on, 2013, pp. 960-969. doi:10.1109/ICPP.2013.114.

[13] T. Miller, X. Pan, R. Thomas, N. Sedaghati, R. Teodorescu, Booster: Reactive core acceleration for mitigating the effects of process variation and application imbalance in low-voltage chips, in: High Performance Computer Architecture (HPCA), 2012 IEEE 18th International Symposium on, 2012, pp. 1-12. doi:10.1109/HPCA.2012.6168942.

[14] J. Lee, V. Sathisha, M. Schulte, K. Compton, N. S. Kim, Improving throughput of power-constrained gpus using dynamic voltage/frequency and core scaling, in: Parallel Architectures and Compilation Techniques (PACT), 2011 International Conference on, 2011, pp. 111-120. doi: 10.1109/PACT.2011.17.

[15] P. G. Bridges, K. B. Ferreira, M. A. Heroux, M. Hoemmen, Fault-tolerant linear solvers via selective reliability, ArXiv e-printsarXiv:1206.1390.

[16] M. Heroux, R. Bartlett, V. H. R. Hoekstra, J. Hu, T. Kolda, R. Lehoucq, K. Long, R. Pawlowski, E. Phipps, A. Salinger, H. Thornquist, R. Tuminaro, J. Willenbring, A. Williams, An Overview of Trilinos, Tech. Rep. SAND2003-2927, Sandia National Laboratories (2003).

[17] J. Elliott, M. Hoemmen, F. Mueller, Evaluating the impact of sdc on the gmres iterative solver, in: Proceedings of the 2014 IEEE 28th International Parallel and Distributed Processing Symposium, IPDPS '14, IEEE Computer Society, Washington, DC, USA, 2014, pp. 1193-1202. doi:10.1109/IPDPS.2014.123.

URL http://dx.doi.org/10.1109/IPDPS.2014.123 
[18] M. Snir, R. W. Wisniewski, J. A. Abraham, S. V. Adve, S. Bagchi, P. Balaji, J. Belak, P. Bose, F. Cappello, B. Carlson, A. A. Chien, P. Coteus, N. DeBardeleben, P. C. Diniz, C. Engelmann, M. Erez, S. Fazzari, A. Geist, R. Gupta, F. Johnson, S. Krishnamoorthy, S. Leyffer, D. Liberty, S. Mitra, T. Munson, R. Schreiber, J. Stearley, E. V. Hensbergen, Addressing failures in exascale computing, IJHPCA (2014) 129-173.

[19] J. Elliott, M. Hoemmen, F. Mueller, A numerical soft fault model for iterative linear solvers, in: Proceedings of the 24th International Symposium on High-Performance Parallel and Distributed Computing, HPDC '15, ACM, New York, NY, USA, 2015, pp. 271-274. doi:10.1145/2749246.2749254. URL http://doi .acm .org/10.1145/2749246.2749254

[20] J. J. Elliott, M. F. Hoemmen, F. Mueller, Tolerating Silent Data Corruption in Opaque Preconditioners., 2014.

[21] D. Zhu, R. Melhem, D. Mosse, The effects of energy management on reliability in real-time embedded systems, in: Computer Aided Design, 2004. ICCAD-2004. IEEE/ACM International Conference on, 2004, pp. 35-40. doi:10.1109/ICCAD . 2004.1382539

[22] D. Zhu, R. Melhem, D. Mosse, E. Elnozahy, Analysis of an energy efficient optimistic tmr scheme, in: Parallel and Distributed Systems, 2004. ICPADS 2004. Proceedings. Tenth International Conference on, 2004, pp. 559-568. doi:10.1109/ICPADS.2004.1316138.

[23] V. Chandra, R. Aitken, Impact of technology and voltage scaling on the soft error susceptibility in nanoscale cmos, in: Defect and Fault Tolerance of VLSI Systems, 2008. DFTVS '08. IEEE International Symposium on, 2008, pp. 114-122. doi:10.1109/DFT.2008.50.

[24] L. Tan, S. L. Song, P. Wu, Z. Chen, R. Ge, D. J. Kerbyson, Investigating the interplay between energy efficiency and resilience in high performance computing, in: 2015 IEEE International Parallel and Distributed Processing Symposium, 2015, pp. 786-796. doi:10.1109/IPDPS.2015.108 